\title{
Interaction Dynamics in a Social Network Using Hidden Markov Model
}

\author{
Davis Bundi Ntwiga, Carolyne Ogutu \\ School of Mathematics, University of Nairobi, Nairobi, Kenya \\ Email: dbundi@uonbi.ac.ke
}

How to cite this paper: Ntwiga, D.B. and Ogutu, C. (2018) Interaction Dynamics in a Social Network Using Hidden Markov Model. Social Networking, 7, 147-155. https://doi.org/10.4236/sn.2018.73012

Received: May 8, 2018

Accepted: July 15, 2018

Published: July 18, 2018

Copyright $\odot 2018$ by authors and Scientific Research Publishing Inc. This work is licensed under the Creative Commons Attribution International License (CC BY 4.0).

http://creativecommons.org/licenses/by/4.0/

\begin{abstract}
Agents interactions in a social network are dynamic and stochastic. We model the dynamic interactions using the hidden Markov model, a probability model which has a wide array of applications. The transition matrix with three states, forgetting, reinforcement and exploration is estimated using simulation. Singular value decomposition estimates the observation matrix for emission of low, medium and high interaction rates. This is achieved when the rank approximation is applied to the transition matrix. The initial state probabilities are then estimated with rank approximation of the observation matrix. The transition and the observation matrices estimate the state and observed symbols in the model. Agents interactions in a social network account for between $20 \%$ and $50 \%$ of all the activities in the network. Noise contributes to the other portion due to interaction dynamics and rapid changes observable from the agents transitions in the network. In the model, the interaction proportions are low with $11 \%$, medium with $56 \%$ and high with $33 \%$. Hidden Markov model has a strong statistical and mathematical structure to model interactions in a social network.
\end{abstract}

\section{Keywords}

Agents, Interactions, Social Network, Hidden Markov Model, Singular Value Decomposition

\section{Introduction}

A social network is a set of people, each of whom is acquainted with some subset of the others. This set of people is referred to as agents. The interactions of agents form a social network where they link and stochastically evolve over time [1]. Evolution has history of past and current interactions that contain the information about the agent abilities and dispositions [2]. The social interactions 
affect user activity and pairs of individuals with strong ties are likely to exhibit greater similarity than those with weak ties [3]. The social interactions evolve due to agents strategies that are random in order to reinforce the benefits gained. The evolution can lead to decay, forgetting or reinforcement of existing interactions [4] [5].

Networks have vast amount of social networking data necessary to understand the properties and the behavior of social networks [1] [6] [7] [8]. Human interactions study found that the use of networks was important as interactions were dynamic and this had an impact on the properties exhibited by the network evolution [8]. The dynamics, complexities and stochasticity in the network interactions can be captured through Hidden Markov Model (HMM). An analysis of HMM shows that the model is adaptable to a wide array of applications due to its strong statistical foundation; evaluation and training algorithms; ability to handle new data robustly and predict similar patterns efficiently [9]. A number of models of social network interactions have been studied based on different forms and structures [1] [5] [10] [11] [12] [13] [14].

In this paper, we seek to enhance research in this area of interactions in social networks by introducing the use of HMM. We found no similar work that has used HMM and singular value decomposition (SVD) in social network interaction studies. We model social network interactions with a three state transition matrix: forgetting, reinforcement and exploration processes as they drive the dynamics of the network [15]. The observation matrix outputs are low, medium and high interaction levels and the initial state probabilities for the social dynamics are estimated using SVD technique.

\section{Dynamic Interactions}

Agents in a network make friends on asymmetric weights. The choice is by chance determined by the relative weight each agent has assigned to the other agents. The dynamics of friendships have interactions that are random in which social network structure emerges from the stochastically evolving social networks [5]. A way to improve on the model of social dynamics is through the introduction of the interaction contextual information. The information was incorporated to reflect the agents interaction dynamics better. Information theory is used to select features that generate a compact and effective feature vector for each agent interaction [16]. Social effects in an interaction model using HMM are introduced where the effects influence a user's activity based on the activity of a user's neighbor thus increasing the model explanatory and predictive power [2]. Static interactions of facebook users were modeled and analyzed using graphs. Decay in the rate and amount of interactions between different pairs of users in facebook were observed over time [17]. Activities in a social network are a sequence of events where [11] shows that social relationships changes continuously in a way correlated with the dynamical processes taking place during social interactions. A generative model based on forgetting, reinforcement and 
exploration processes in online social network is undertaken where a user has a forgetting behavior that shows decline in pairs of user attraction. A user counteracts this by exhibiting a reinforcing behavior to keep the important relationships alive. Also, each user has an exploring behavior to continuously explore new interaction relationships [15].

Studies involving the use of HMM for varied applications due to its flexible general purpose approach to modeling dynamical systems are presented. HMM do not have a limitation for their applications [18]. HMM modeled trust in a dynamic environment that changes with time [16]. A probabilistic approach used the HMM to model a social network dynamics [2]. The agents' dynamics behavior feedback mechanism was modeled using HMM to enhance the trust and reputation evaluations about the trustee [19]. A HMM approach and SVD estimated trust levels by extracting reputation ratings from the network matrix and a $20 \%$ discounting with SVD is found to provide an optimal trust levels in a social network [6].

We model the interaction dynamics of users in a social network using HMM with simulation data. The transition matrix has three processes of forgetting, reinforcement and exploration [15]. The observation matrix is estimated from the transition matrix using the matrix factorization method [20]. The initial state probabilities are estimated from the errors of the matrix factorization method when estimating the observation matrix.

\section{Hidden Markov Model}

The characterization of HMM is outlined [1] [21].

- The number of states $(M=3)$ in the model with the set of states denoted as $S=\left\{S_{1}, S_{2}, S_{3}\right\}=\{F, R, E\}=\{$ Forgetting, Reinforcement, Exploration $\}$.

- Thestatetransition probability distribution $A=\left\{a_{i j}\right\}$, where $a_{i j}=P\left[q_{t+1}=S_{j} \mid q_{t}=S_{i}\right]$, where $1 \leq i, j \leq M, t=1, T$.

- The number of distinct observation symbols $(K=3)$ per state. We denote the set of observation symbols corresponding to the physical output of the system being modeled as $V=\left\{v_{1}, v_{2}, v_{3}\right\}=\{$ Low, Medium, $\operatorname{High}\}=\{L, M, H\}$.

-Theobservation symbol probability matrix $B=\left\{b_{j}(k)\right\}$, where $b_{j}(k)=P\left[v_{k} \mid S_{j}\right], 1 \leq j \leq M, 1 \leq k \leq K$.

- Theinitial state probability vector $\pi=\left\{\pi_{i}\right\}$, where $\pi=P\left[q_{1}=S_{i}\right], 1 \leq i \leq M$.

- Observation sequence $O=O_{1}, O_{2}, \cdots, O_{T}$, where each observation $O_{t}$ is one of the symbols from $V$ and $T$ is the number of observations in the sequence.

A complete set of parameters of the HMM model is represented as $\lambda=(A, B, \pi)$. The social interaction processes of forgetting, reinforcement and exploration that drives dynamics of social interactions over the network forms a Markov process which is the basis for our Hidden Markov transition matrix $A$. We assume that an agent can transit from one state to another based on transition probabilities. The observation matrix $B$ is estimated using SVD by elimi- 
nating errors in matrix $A$ while the initial state probabilities are estimated from the errors eliminated while estimating the observation matrix. The transitions when the noise component was reduced led to the observation of the actual interaction dynamics exhibited by the agents. Noise in the observation matrix represents the component that does not enhance the interactions as it is difficult to decode in the formation of relationships. The decision of who to interact or not interact with increases the noise component in the transition and observation matrix due to the uncertainty of the agent's behavior.

\section{User Interaction Analysis}

The rate of user interactions across different social networks has been highlighted by different research work. Nearly all interactions can be attributed to only the top $5 \%$ of the users in facebook [17]. 30\% of facebook user's pairs interact consistently from one month to the next. Interaction edges only accounts for $32 \%$ of the total edges, meaning users only interact with a small subset of their friends. Nearly $78 \%$ of interactions account for total wall posts which represents the friendship maintenance effort [15].

We conclude that reinforcement is at $32 \%$, exploration at $46 \%$ and forgetting is at $22 \%$ of all the user activities in a social network. These form the main diagonal of the transition matrix and the other real valued entries of the matrix are derived through simulation. This approach is applied due to lack of real life social networking data. The study varied the entries of the main diagonal of the transition matrix at the analysis level.

\section{Singular Value Decomposition}

Matrix approximation technique is widely researched and a common tool used heavily in recommendation systems, bioinformatics, computer vision and text processing. SVD is a matrix factorization method that has been used widely in different applications ever since an efficient algorithm for its computation was developed [20]. SVD is a stable and effective method to split the system into a set of linearly independent components, each of them bearing its own energy contribution [22].

The SVD eliminated the noise in the transition matrix $A$ to estimate the observation matrix. The noise in the transition and observation matrices is eliminated by adopting an accuracy threshold level of $\alpha$. A reduced rank approximation with error of $1-\alpha$ of matrix $A$ to represent matrix $B$ is selected. Let $\hat{A}$ be the matrix for the retained $\alpha$ and $\tilde{A}$ be the matrix for the eliminated $1-\alpha$ of matrix $A$. The term error in SVD is defined by [23] as:

$$
\hat{\alpha}=1-\sqrt{\frac{\sum_{i=1}^{k} \sigma_{i}^{2}}{\sum_{i=1}^{N} \sigma_{i}^{2}}}
$$

The relative error is estimated for a sum of the first terms of the SVD outer product expansion [20] [23]. SVD is used for optimal low rank approximation and 
a partial SVD can be used to construct a rank $k$ approximation. The retained $\alpha$ of the singular values of matrix $A$ are reconstructed to estimate matrix $\hat{A}$ which is normalized to estimate the observation matrix $B$. The initial state probability vector is then estimated from matrix $\tilde{A}$ of $1-\alpha$ of the singular value of matrix $A$ that was eliminated as noise, due to uncertainty in the agents interactions.

\section{Results and Discussions}

This section highlights the results and discussions from the agents interactions in a social network.

Table 1 shows the descriptive statistics for two samples generated with two random generators. Each sample has two parts, the first $40 \%$ and the second $30 \%$ with the error estimation in each case. When compared, the first section of each of the two samples has higher variability as compared to the second part of each sample. This is due to changes observable in the social interactions as agents' interactions in a social network account for around $50 \%$ of social dynamics.

In Table 2, the observation probabilities increased from low to high when interaction rates ranges between $10 \%$ and $50 \%$. The interactions intensity is higher in the high interactions rate for the first partition compared to the second partition. Thus, the most active proportion of agents' is estimated at around $50 \%$.

In Figure 1, the determinants of the product between the observation and transition matrices with different rank approximation are plotted. The figure shows the changes observable in a social network in terms of interdependence and independence of the agents interactions. At $10 \%$ to $50 \%$ interaction rate, the matrix product has a determinant of zero. This high level of dependency amongst the agents shows high levels of interactions. This dependency through the singular matrix as a product of the transition and observation matrices shows that the activities of an agent can be expressed as a linear combination of the other agents in the network. The interactions are intense and agents actively change their positions during these times to achieve their desired relationship levels. This slows down from the error estimation above $50 \%$ where independence

Table 1. Descriptive statistics and the interaction rates.

\begin{tabular}{ccccc}
\hline & \multicolumn{2}{c}{ Sample 1 } & \multicolumn{2}{c}{ Sample 2 } \\
\hline Statistic & $10 \%-50 \%$ & $60 \%-90 \%$ & $10 \%-50 \%$ & $60 \%-90 \%$ \\
\hline Mean & 0.5322 & 0.5040 & 0.5973 & 0.4587 \\
Standard deviation & 0.1158 & 0.0524 & 0.1818 & 0.0807 \\
Coefficient of variation & 21.7587 & 10.3968 & 30.437 & 17.5932 \\
\hline
\end{tabular}

Table 2. Observation probabilities and interaction rates.

\begin{tabular}{cccc}
\hline Interaction rates & Low & Medium & High \\
\hline $10 \%-50 \%$ & 0.2510 & 0.3403 & 0.4087 \\
$60 \%-90 \%$ & 0.3218 & 0.3231 & 0.3551 \\
\hline
\end{tabular}




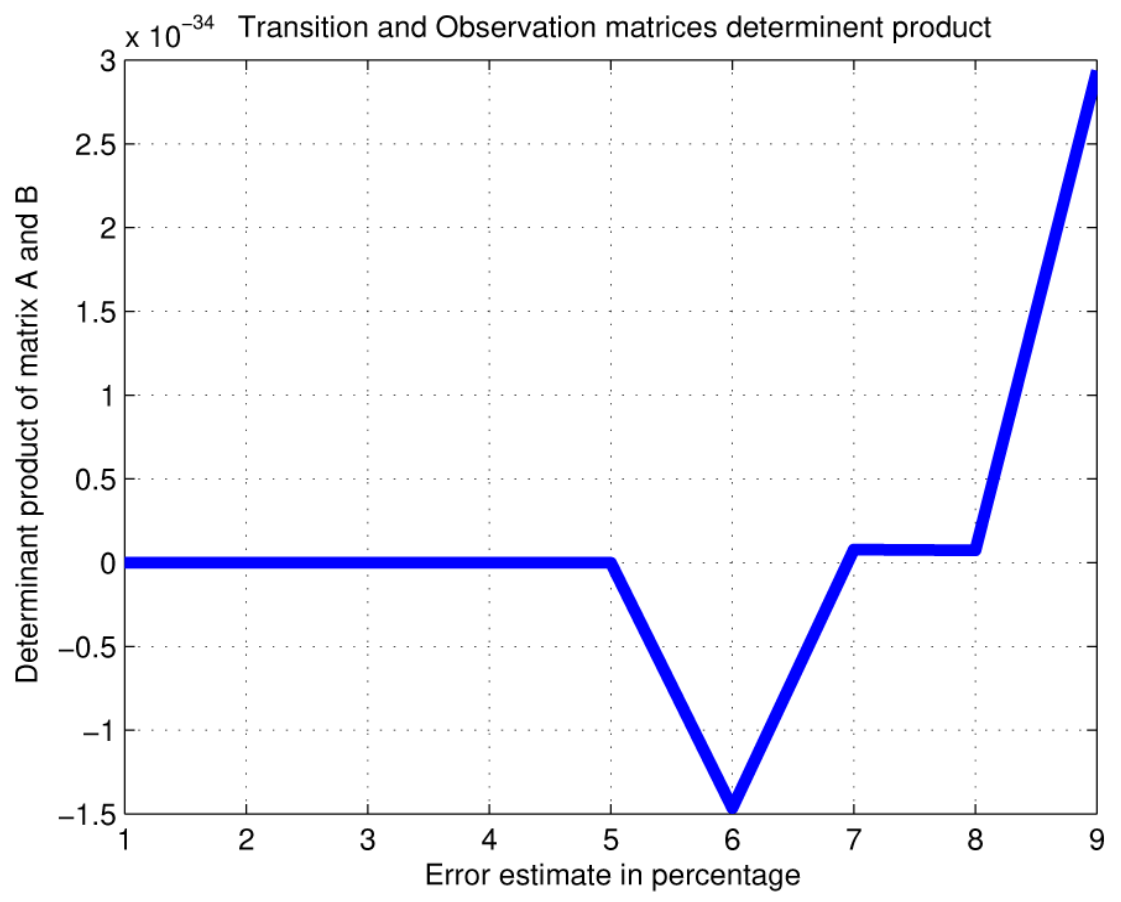

Figure 1. Dynamics of the determinant of the product between the observation and transition matrices.

levels increases.

Figure 1 and Table 1 highlight that agents are more active in changing positions as they interact with the interaction rates of between $10 \%$ and $50 \%$. Table 1 has high variability estimated by the coefficient of variation. Above $60 \%$, low levels of interdependency are exhibited, an indication of minimum interaction rates in the social network.

In Figure 2, the medium and high interaction rates were observable with the estimate error of between $10 \%$ and $50 \%$. At $60 \%$, the interaction rates falls to low and then increases again to medium and high as estimation error increases. This compares well with Figure 1 of the singular matrix emitted by the transitions and observations of the agents. Further observations from Figure 2 is that low interactions accounts for $11 \%$ of all the activities, medium interactions with $56 \%$ and high interaction rates accounts for $33 \%$. We used simulation but similar observations were noted [3] [17] [24]. Agents' reinforce and explore new interactions at the start and this dips and increases again with time. A general trend in a social network is for the agents to actively pursue new relationships, some waning with time, others being reinforced and new ones being formed. The other component of the social network is the noise due to these dynamic changes in the network.

\section{Conclusion}

In our model, agents interactions from the HMM and SVD estimates showed $11 \%$ low observations, $56 \%$ medium interaction rates and $33 \%$ high interactions 


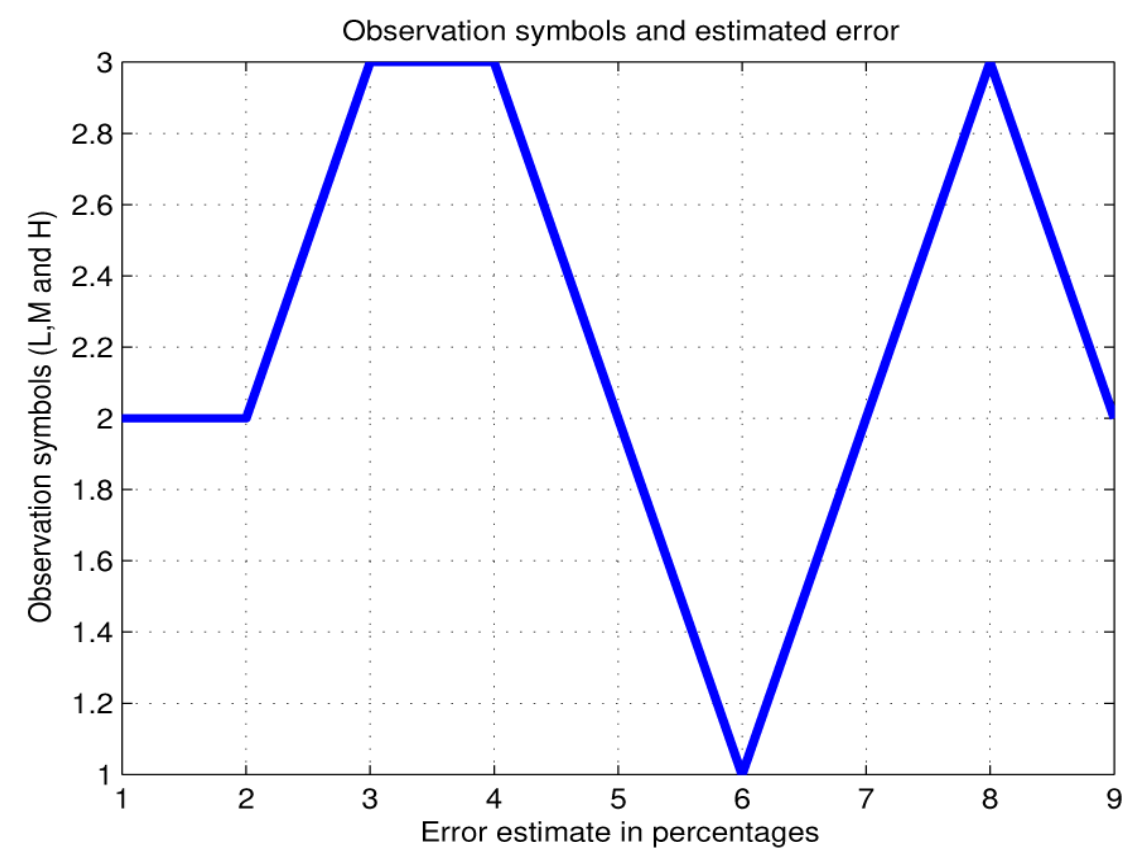

Figure 2. Observation symbols $(L=1, M=2$ and $H=3$ ) versus the estimated errors.

observed. The interaction rate of medium and high is observed within the first $50 \%$ of error estimation as the other component is noise in the network due to interaction dynamics and rapid changes from the agents activities. The SVD is an ideal method to estimate the observation matrix from the transition matrix and the initial probabilities from the observation matrix. The HMM is a technique that can model interaction dynamics in a social network due to its adaptability to model stochastic processes.

\section{References}

[1] Ntwiga, D.B. (2016) Social Network Analysis for Credit Risk Modeling. Unpublished Ph.D. Thesis, School of Mathematics, University of Nairobi, Nairobi.

[2] Raghavan, V., Steeg, G., Galstyan, A. and Tartakovsky, A.G. (2014) Modeling Temporal Activity Patterns in Dynamic Social Networks. IEEE Transactions on Computational Social Systems, 1, 89-107. https://doi.org/10.1109/TCSS.2014.2307453

[3] Xiang, R., Neville, J. and Rogati, M. (2010) Modeling Relationship Strength in Online Social Networks. Proceedings of the 19th International Conference on World Wide Web, Raleigh, 26-30 April 2010, 981-990. https://doi.org/10.1145/1772690.1772790

[4] Meyers, R.A. (2009) Complex Systems in Finance and Economics (Selected Entries from the Encyclopedia of Complexity and Systems Science). Springer, New York.

[5] Skyrms, B. and Pemantle, R. (2000) Dynamic Model of Social Network Formation. Proceedings of the National Academy of Sciences of the United States of America, 97, 9340-9346. https://doi.org/10.1073/pnas.97.16.9340

[6] Ntwiga, D.B., Weke, P. and Kirumbu, M.K. (2016) Trust Model for Social Network using Singular Value Decomposition. Interdisciplinary Description of Complex Systems, 14, 296-302. https://doi.org/10.7906/indecs.14.3.2 
[7] Netrvalova, A. and Safarik, J. (2011) Trust Model for Social Network. 2011 IEEE 10 th International Conference on Trust, Security and Privacy in Computing and Communications, Changsha, 16-18 November 2011, 102-107.

[8] Pupazan, E. (2011) Social Networking Analytics. BMI paper, Vrije University Amsterdam, Amsterdam.

[9] Dymarski, P. (2011) Hidden Markov Models, Theory and Applications. Intech Open Access. http://www.intechopen.com https://doi.org/10.5772/601

[10] Starnini, M., Baronchelli, A. and Romualdo, P. (2013) Modeling Human Dynamics of Face-to-Face Interaction Networks. Physical Review Letters, 110, Article ID: 168701. https://doi.org/10.1103/PhysRevLett.110.168701

[11] Zhao, K., Stehle, J., Bianconi, G. and Barrat, A. (2011) Social Network Dynamics of Face-to-Face Interactions. Physical Review E, 83, Article ID: 056109. https://doi.org/10.1103/PhysRevE.83.056109

[12] Gonzalez, M.C., Lind, P.G. and Herrmann, H.J. (2006) Model of Mobile Agents for Sexual Interactions Networks. The European Physical Journal B-Condensed Matter and Complex Systems, 49, 371-376. https://doi.org/10.1140/epjb/e2006-00068-2

[13] Ntwiga, D.B., Weke, P., Manene, M. and Mwaniki, J. (2016) Modeling Trust in Social Network. International Journal of Mathematical Archive, 7, 64-68.

[14] Resnick, P., Zeckhouse, R., Friedman, E. and Kuwabara, K. (2000) Reputation System. Communications of the Association of Computing Machinery, 43, 45-48. https://doi.org/10.1145/355112.355122

[15] Yang, Z., Xue, J., Wilson, C., Zhao, B.Y. and Dai, Y. (2015) Uncovering User Interaction Dynamics in Online Social Networks. Proceedings of the 9 th International Association for the Advancement of Artificial Intelligence (AAAI) Conference on Web and Social Media, Oxford, 26-29 May 2015, 698-701.

[16] Liu, X. and Datta, A. (2012) Modeling Context Aware Dynamic Trust Using Hidden Markov Model. Proceedings of the 26th AAAI Conference on Artificial Intelligence, Toronto, 22-26 July 2012, 1938-1944.

[17] Wilson, C., Boe, B., Sala, A., Puttaswamy, K.P.N. and Zhao, B.Y. (2009) User Interactions in Social Networks and Their Implications. Association of Computing Machinery, New York.

[18] Bilmes, J.A. (2006) What Hidden Markov Models Can Do. IEICE Transactions, Information and Systems, E89-D, 869-891. https://doi.org/10.1093/ietisy/e89-d.3.869

[19] Ehab, E. and Sassone, V. (2013) A HMM Based Reputation Model. Advances in Security of Information and Communication Networks, 381, 111-121.

[20] Musco, C. and Musco, C. (2015) Stronger and Faster Approximate Singular Value Decomposition via Block Lanczos Method. arXiv:1504.05477v2

[21] Rabiner, L.R. (1989) A Tutorial on Hidden Markov Models and Selected Applications in Speech Recognition. Proceedings of the IEEE, 77, 257-286. https://doi.org/10.1109/5.18626

[22] Lee, J., Kim, S., Lebanon, G. and Singer, Y. (2013) Local Low-Rank Matrix Approximation. Proceedings of the 30 th International Conference on Machine Learning, Atlanta, 16-21 June 2013, 82-90.

[23] Kalman, D. (1996) A Singularly Valuable Decomposition: The SVD of a Matrix. The College Mathematics Journal, 27, 1-23.

https://doi.org/10.1080/07468342.1996.11973744 
[24] Viswanath, B., Mislove, A., Cha, M. and Gummadi, K.P. (2009) On the Evolution of User Interaction in Facebook. Association of Computing Machinery, New York. $\underline{\text { https://doi.org/10.1145/1592665.1592675 }}$ 